Results $56.9 \%$ children were males and mean age was 7.4 years. Of the ten main differential diagnoses, burn and long bone fractures frequently caused severe pain ( $80.8 \%$ and $50.9 \%$ respectively). 152 (25\%) children with pain did not receive analgesia (39\% of children with mild pain, $22 \%$ moderate pain and $15 \%$ severe pain; $\mathrm{P}=0.0001$ ). For children with severe pain, $66.0 \%$ received simple analgesia $(\mathrm{P}=0.001)$ and only $38.5 \%$ received opioids $(\mathrm{P}=0.0001)$ with younger children ( $0-5$ years old) receiving less intravenous diamorphine and more oral morphine than older children $(13-18)(14 \%$ vs $52 \%$ and $46.0 \%$ vs $30.0 \%$ respectively; both $\mathrm{P}=0.0001)$. None was given intranasal diamorphine.

Conclusions Children with pain were not adequately analgised, especially those with severe pain and those that were younger. Children coming with conditions known to be severely painful (i.e. burn and long-bone fractures) should be considered for opioids regardless of their pain scores and opioid of choice is intranasal diamorphine, requiring no intravenous access with similar efficacy as the more common intravenous morphine.

\section{HEART RATES AND RESPIRATORY RATES ARE ASSOCIATED WITH MANCHESTER PAIN SCORES IN CHILDREN PRESENTED AT THE EMERGENCY DEPARTMENT}

doi:10.1136/archdischild-2012-302724.1621

${ }^{1} \mathrm{~N}$ Seiger, ${ }^{1} \mathrm{M}$ van Veen, ${ }^{2} \mathrm{EW}$ Steyerberg, ${ }^{3} \mathrm{~J}$ van der Lei, ${ }^{4} \mathrm{M}$ Ruige, ${ }^{4} \mathrm{AHJ}$ van Meurs, 5 Maconochie, 'HA Moll. 'General Paediatrics, Erasmus MC - Sophia Children's Hospital; ${ }^{2}$ Public Health; ${ }^{3}$ Medical Informatics, Erasmus MC, Rotterdam; ${ }^{4}$ General Paediatrics, Haga Hospital - Juliana Children's Hospital, The Hague, The Netherlands, ${ }^{5}$ Paediatric Accident and Emergency, St. Mary's Hospital, London, UK

Background and Aims Acute pain increases vital signs and is measured by the Manchester pain scale during triage. This multicentre observational study aims to determine associations between respiratory rates or heart rates and Manchester pain scores and to derive age and pain appropriate centiles for children presenting at emergency departments (EDs).

Methods Triaged children ( $<16$ years) presented at EDs in Rotterdam between 2006 and 2010, in The Hague between 2006 and 2007, and in London in 2010, were included. Pain scores were obtained by the Manchester painscale (range 0-10). This painscale combines a visual analogue scale, a verbal descriptor scale, and a pain behaviour tool. Univariate and multivariable regression analyses were performed. Secondly, pain and age appropriate heart rate and respiratory rate centile charts were created.

Results We included 45344 children. In multivariable analysis, the average heart rate of children with mild or moderate pain decreased significantly with 6.1 (95\%CI5.2-6.9) and 5.0 (95\%CI3.9-6.1) beats per minute respectively, while children with severe pain had increased heart rates $(6.5 .95 \%$ CI4.5-8.6) when compared with children without pain. Centile charts for children younger than twelve years showed increased heart rates for children with severe pain. This effect disappeared in older children. The association between respiratory rates and pain scores showed similar trends as heart rate centiles, but the change in respiratory rates was small.

Conclusion New derived centile charts for children are available to interpreted heart rates and respiratory rates in relation to pain. In younger children, heart rates increased due to severe pain.

\section{THE EVALUATION OF ANXIETY AND PAIN RELATED TO DENTAL TREATMENT IN CHILDREN UNDER THE AGE OF FIVE YEARS (BRAZIL)}

doi:10.1136/archdischild-2012-302724.1622

${ }^{1} \mathrm{E}$ Costa, ${ }^{2} \mathrm{C}$ Costa, ${ }^{1} \mathrm{RE}$ Adamos. ${ }^{1} \mathrm{Clinical}$ Research, Costa Odontologia Integrada; ${ }^{2}$ Dental Radiology, University of Sao Paulo, Sao Paulo, Brazil
The Aim of this study was to evaluate anxiety and pain related to dental treatment in children under the age of five years. This cross sectional study was carried out with 350 children of both sexes. Socioeconomic data, dental anxiety and dental pain experience, as well as the assessment of the child's oral health status, were obtained through a questionnaire answered by the child's parent or guardian. Dental anxiety was measured using the Dental Anxiety Question (DAO). The prevalence of dental anxiety was $22.9 \%$ and that of dental pain was $6.8 \%$. There was an association between these two variables $(p<0.0001)$. There was also an association between dental pain, age, family income and assessment of oral health status. The poorest rating of the child's oral health and the lowest family income were correlated with the highest percentages of a history of dental pain. Dental anxiety was related to a history of dental pain in children under the age of five years.

\section{PAIN AFTER CIRCUMCISION: COMPARISON OF CONVENTIONAL AND PLASTIC CLAMP CIRCUMCISIONS}

doi:10.1136/archdischild-2012-302724.1623

F Senel, F Misirlioglu. Dr. Sami Ulus Hospital, Ankara, Turkey

Background and Aims In the current study we compared the intensitiy of post-circumcision pain among the male children circumcised by plastic Ali Klamp and conventional dissection technique.

Method Study included 126 children with a mean age of $9.2 \pm 2.8$ years circumcised by plastic Alis klamp technique, and 114 children with a mean age of $9.5 \pm 2.4$ years circumcised by conventional dissection technique under local anesthesia. A visual pain scale chart was utilized to asses the intensitiy of post-circumcision pain.

Results The initial pain scores were similar among both group of males which initiated $4.2 \pm 1.4$ hours following circumcision. The mean pain scores at 8 (4.34 vs. 5.8; $\mathrm{p}<0.002), 12$ (3.12 vs. 5.02; $\mathrm{p}<0.001)$, and 18 (2.4 vs. $4.01 ; \mathrm{p}<0.001)$ hours were significantly lower among the males circumcised by plastic clamp technique compared to those circumcised by conventional technique. Intensity of pain was similar in both groups after 24 hours following circumcisions.

Conclusion Circumcision performed by plastic Alis klamp technique in male children is associated with a lower intensity of pain compared to circumcisions performed by conventional dissection technique. We suggest the utilization of this technique as it provides a a better post-circumcision period compared to conventional technique particularly in children who are afraid of circumcision as a matter of fact.

\section{THE COMPARISON OF THE EFFECTS OF MASSAGING AND ROCKING ON INFANTILE COLIC}

doi:10.1136/archdischild-2012-302724.1624

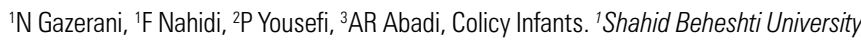
of Medical Sciences, Tehran: ${ }^{2}$ Arak Medical University, Amir Kabir Hospital, Arak ${ }^{3}$ Statistic Department, Shahid Beheshti University of Medical Sciences, Tehran, Iran

Background and Aims The aim of this study was to compare the effects of massaging and rocking on the treatment of infantile colic. Methods This randomized clinical trial involved 100 infants $<13$ weeks of age who were considered colic were randomly assigned to either infant massage $(n=50)$ or rocking groups $(n=50)$, in Arak, Iran, in 2010. In the massage group, trained individuals taught the infant massage technique to the parents and gave them a brochure. Daily interventions were recommended in both groups 3 times daily for one week. Parents recorded infant crying times, duration, and severity over a week. After one week, data were analyzed by SPSS and statistical significance tests $(\mathrm{P}<0.05)$. 
Results Two groups did not differ significantly in infant and mother demographic information. After one week of intervention, the mean difference of total crying time, duration and severity were 4.08 (1.83) time/day, 2.81 (1.77) hour/day and 2.9 (2.37) in massage group and 0.56 (2.28) time/day, 0.27 (1.09) hour/day and 0.02 (1.64) in vibrating group, respectively. The mean of total crying time, duration and severity decreased in both groups but there was a more significant reduction in crying times, duration and severity in massage group than the rocking group.

Conclusions Our findings demonstrated that infant massage was effective in reducing the time, duration, and severity of crying in colicky infants.

\section{PAIN IN CHILDREN: KNOWLEDGE AND PERCEPTIONS OF NURSING STAFF AT A RURAL TERTIARY CARE TEACHING HOSPITAL IN WESTERN INDIA}

doi:10.1136/archdischild-2012-302724.1625

${ }^{1} \mathrm{AS}$ Nimbalkar, ${ }^{1} \mathrm{AR}$ Dongara, ${ }^{2} \mathrm{JD}$ Ganjiwale, ${ }^{1,2}$ SM Nimbalkar. 'Department of Pediatrics, Pramukhswami Medical College; ${ }^{2}$ Central Research Services, Charutar Arogya Mandal, Anand, India

Backgrounds and Aims Pain is a common cause for which patients seek treatment and an unpleasant side effect of our treatment. Young children are not able to express their pain. It is the care-givers responsibility to assess and manage their pain. Caregivers perception of the child's pain depends on various factors. We studied the perception and knowledge regarding pain amongst nursing staff at our centre. We compared these across three groups as per their exposure to pediatric patients (routinely, occasionally or rarely exposed to pediatric patients).

Method Consensually validated questionnaire containing combination of questions from basic (must know) and advanced (nice to know) areas of knowledge about nursing pediatric patients and questions related to nurses' perception about pain in pediatric patients was administered to eligible nursing staff at Rural Tertiary Care Hospital in Western India. The responses were analyzed using descriptive statistics and comparisons were made by chi-square test. Result 351 usable questionnaires (83.37\%) out of 421 were returned. The knowledge of the nurses regarding pain was observed to be poor. $60 \%$ of all the nurses had complete knowledge of all the basic questions asked. Only $3.1 \%$ had answered all of the five advanced questions correctly, while $96.9 \%$ of the nurses had answered one or more questions incorrectly.

Conclusions The deficit in knowledge and shortcomings in perception needs to be addressed and steps need to be taken to improve the nurse's knowledge and modify beliefs and attitude of the nursing staff towards the pain of the pediatric patients.

\section{IMPROVING PAIN MANAGEMENT IN TERTIARY PEDIATRIC HOSPITAL- HIGH HOPES AND ORDINARY PROBLEMS}

doi:10.1136/archdischild-2012-302724.1626

J Kalbowiak, M Manowska, P Łaniewski- Wołk, E Pietraszek- Jezierska. Anaethesia and Intensive Care, Children's Memorial Health Institute, Warszawa, Poland

Background and Aims Observation of pain management problems in tertiary pediatric hospital (Children's Memorial Health Institute, Warsaw, Poland) resulted in anaesthesia and intensive care team initiative of pain practice improvement.

Methods In November 2011 Pain Treatment Committee represented by 22 doctors and 20 nurses of all hospital departments was established.

In November/December 2011 pilot training programme for nurses of Pediatric Urology, Neurosurgery and Cardiac Surgery Departments was performed.
In December 2011 written pain assessment and treatment guidelines accepted by the committee members and hospital administration where announced. They included:

- obligatory pain intensity assessment with age/communication skills- appropriate tools (NIPS, FLACC, Wong- Baker, VAS).

- pain treatment adequate to individual pain intensity with multimodal analgesia use.

- restriction in muscular injections of analgesics.

- analgesics dosing guidelines.

- perioperative analgesia algorithms based on predicted pain intensity.

Between February and March 2012201 practitioners and 391 nurses participated in pain assessment and management seminars.

Results Preliminary report on analgesia practice in surgical units revealed:

- poor compliance with pain assessment guidelines

- $100 \%$ reduction in intramuscular opioid use in one of the departments.

- improvement in analgesic prescriptions practice with individual variability between practitioners.

- increase in number of pain consultations

- no improvement with use of regional analgesia, insufficient number of PCA pumps.

Conclusions During few months after introducing hospital pain management guidelines we notice a change in pain practice but many problems still exist. Analgesia quality improvement is longterm process requiring multidisciplinary approach.

\section{APPLICATION OF PULSE OXIMETRY FOR DETERMINING THE STATUS OF THE TOOTH IN CHILDREN}

doi:10.1136/archdischild-2012-302724.1627

H Jafarzadeh, N Dastmalchi. Mashhad University of Medical Sciences, Mashhad, Iran

Tests relying on the passage of the light through a tooth have been considered to be a suitable means of assessing pulp vitality in children. Pulse oximetry which is an effective, objective oxygen saturation monitoring technique broadly used in medicine for recording blood oxygen saturation levels, can also be used in endodontic diagnosis for differential diagnosis of vital and necrotic pulps in young teeth. In this system, light is passed from a photoelectric diode across the tooth structure into a receptor. The instrument detects changes in absorption in both red and infrared light caused by alteration in tissue volume during the cardiac cycle. However, there are some limitations such as the effect of increased acidity and metabolic rate which cause deoxygenating of hemoglobin and changes in the blood oxygen saturation. Because this test produces no noxious stimuli, children usually accept it more readily than routine methods. In this lecture, principles, indications, limitations, influencing factors, and variations in probe design for dental usage in children would be presented.

\section{OXYGEN PRESCRIBING AND SATURATION TARGETING IN NEONATAL INTENSIVE CARE}

doi:10.1136/archdischild-2012-302724.1628

R Cronin, J Behrsin, P Bustani. Neonatal Unit, Jessop Wing, Sheffield, UK

Background Many newborns require oxygen; this should be prescribed as with other medical gases. In babies due to the risks from both hypoxia and hyperoxia oxygen saturation targeting to provide optimal safe oxygenation is commonly utilized.

Guidelines for the appropriate target saturations and hence monitoring alarm limits have changed locally in light of evidence from the SUPPORT ${ }^{1}$ and BOOST II ${ }^{2}$ trials. 